\title{
Mini review ATF4 and GRP78 as novel molecular targets in ER-Stress modulation for critical COVID-19 patients
}

\author{
Mahtab Shahriari-Felordi ${ }^{1,2} \cdot$ Hani Keshavarz Alikhani ${ }^{2} \cdot$ Seyed-Mohammad Reza Hashemian ${ }^{3} \cdot$ Moustapha Hassan $^{4}$. \\ Massoud Vosough ${ }^{2,4}$ (1)
}

Received: 7 October 2021 / Accepted: 8 December 2021 / Published online: 14 January 2022

(c) The Author(s), under exclusive licence to Springer Nature B.V. 2021

\begin{abstract}
Coronavirus disease 2019 (COVID-19) is caused by severe acute respiratory syndrome coronavirus 2 (SARS-CoV-2) and has resulted in more than 4.4 million deaths worldwide as of August 24, 2021. Viral infections such as SARS-CoV2 are associated with endoplasmic reticulum (ER) stress and also increased the level of reactive oxygen species. Activating transcription factor 4 (ATF4) is preferentially translated under integrated stress conditions and controls the genes involved in protein homeostasis, amino acid transport and metabolism, and also protection from oxidative stress. The GRP78, regulated either directly or indirectly by ATF4, is an essential chaperone in the ER and overexpressed and appears on the surface of almost all cells during stress and function as a SARS-CoV2 receptor. In this mini-review article, we briefly discuss the effects of SARS-CoV2 infection on the ER stress, and then the stress modulator functions of ATF4 and GRP78 as novel therapeutic targets were highlighted. Finally, the effects of GRP78 inhibitory components as potential factors for targeted therapies for COVID-19 critical cases were discussed.
\end{abstract}

Keywords COVID-19 disease · ER stress modulation · ATF4 · GRP78 targeting

\section{COVID-19 infection and ER stress}

The SARS-CoV-2 is a member of Coronaviridae, a kind of enveloped viruses with positive-sense, single-stranded RNA [1]. This virus caused more than 4.4 million deaths worldwide up to August 2021. No specific therapeutic treatment for COVID-19 has been approved so far which highlighting the urgent need to identify new antiviral strategies.

Massoud Vosough

masvos@Royaninstitute.org

1 Department of Molecular Cell Biology-Genetics, Faculty of Basic Sciences and Advanced Technologies in biology, University of Science and Culture, Tehran, Iran

2 Department of Regenerative Medicine, Cell Science Research Center, Royan Institute for Stem Cell Biology and Technology, ACECR, Tehran, Iran

3 Chronic Respiratory Diseases Research Center (CRDRC), National Research Institute of Tuberculosis and Lung Diseases (NRITLD), Shahid Beheshti University of Medical Sciences, Tehran, Iran

4 Experimental Cancer Medicine, Institution for Laboratory Medicine, Karolinska Institutet, Huddinge, Sweden
After infection of cell by this virus, some events happen such as the massive production of viral proteins, virion budding-mediated ER membrane depletion, and the overloading capacity of protein folding lead to ER stress [2]. ER stress induced by three sensors located on the ER membrane including activating transcription factor-6 (ATF6), protein kinase RNA-like endoplasmic reticulum kinase (PERK), and inositol-requiring enzyme-1 $\alpha$ (IRE1 $\alpha$ ) which activates the unfolded protein response (UPR). UPR activation decreases protein synthesis and increases ER folding capacity and restoring cell homeostasis [3]. Thus, pharmacological manipulation of the UPR or other factors involved in the ER stress pathway can be used as a therapeutic strategy against coronavirus infection.

\section{Oxidative stress responses and COVID-19 progression}

Oxidative stress is a physiological state in which the cellular antioxidant buffering capability is overwhelmed by free radicals and eventually causes damage to cellular macromolecules [4]. Oxidative stress can increase the risk of severe 
COVID-19 infection but there is no significant cause-effect relationship between oxidative stress and COVID-19 severity [5]. SARS-CoV-2 infection induces over-activation of immune responses in the infected organs especially lung tissues. Monocytes and macrophages in the infected tissues secret pro-inflammatory cytokines such as IL-1 $\beta$, IL-6, TNF, IL-8 [6]. The lung endothelium is a target for both pro-inflammatory cytokines and the SARS-CoV-2 virus [7]. In response to cytokines, some adhesive molecules such as ICAM1, VCAM1, E-selectin are expressed on the surface of endothelial cells which enhances the adhesion and penetration of leukocytes across the vascular wall into the body tissues. The endothelial cells themselves release pro-inflammatory cytokines and chemokines that recruit immune cells into the site of inflammation. The accumulation of immune cells is accompanied by inflammation and an elevated level of oxidative stress [8]. The high cellular count ratio of neutrophils than lymphocytes in critically ill COVID-19 patients indicated that the excessive levels of reactive oxygen species (ROS) are one of the main causative agents in the host pathological responses [9]. ROS change the tissue homeostasis and induce damages to the red blood cells which contribute to the severity of COVID-19 disease [9]. Therefore, these studies highlighted the strong relationship between oxidative stress and the severity of COVID-19 disease.

\section{ATF4 as ER stress modulator in the cross-talk with oxidative stress}

Several studies have shown a remarkable association between endoplasmic reticulum (ER) stress and oxidative stress [10-12]. However, oxidative stress and ER stress have reciprocal cross-talk; generation of reactive oxygen species (ROS) is not considered a downstream phenomenon for ER stress [13].

The protein kinase RNA-like endoplasmic reticulum kinase (PERK) is one of the first proteins activated in response to ER stress [14]. Dissociation from ER chaperon, $\mathrm{BiP}$, induces the oligomerization and then auto-phosphorylation of PERK. The phosphorylated and activated PERK then phosphorylates the eukaryotic initiation factor $2(\mathrm{eIF} 2 \alpha)$ and the phosphorylated eIF $2 \alpha$ binds tightly and thus inhibited the eIF2B (guanine nucleotide exchange factor). This results the general inhibition of protein synthesis and therefor reduces the protein influx to the endoplasmic reticulum [3]. Activating transcription factor 4 (ATF4) contain small ORF in its $5^{\prime}$ and bypass the eIF $2 \alpha$-dependent translation block. ATF4 is preferentially translated under integrated stress conditions [2]. Imbalance in the normal functions of the PERK and the induction of the activating transcription factor 4 (ATF4) can initiate ROS activation [15]. ATF4 is induced via stress signals, including anoxia/hypoxia, amino acid deprivation, ER stress, and oxidative stress [16]. In normal cells, the reciprocal interaction between these proteins can regulate ROS production through transcriptional regulation [17]. Moreover, while ATF4 controls oxidative stress, its depletion in different organs including liver and lungs causes malfunction and homeostasis impairment $[12,18]$.

Furthermore, it was shown that the GRP78 level is regulated either directly or indirectly by ATF4 [19]. Phosphorylation of eukaryotic initiation factor- $2 \alpha(\mathrm{eIF} 2 \alpha)$ activates ATF4, which regulates the UPR by controlling the expression of UPR target genes that are linked to endoplasmic-reticulum-associated protein degradation (ERAD) and pro-apoptotic CCAAT/enhancer-binding protein (C/ EBP)-homologous protein (CHOP) [19]. In addition, a conserved binding site (5'-TGACGTGA-3') for ATF4 is located upstream to the ER stress response element (ERSE) in the mammalian GRP78 promoter. This binding site is well defined and differ from the C/EBP-ATF composite site that was described previously for the CHOP. Notably, this pathway is not affected by ER-stress so ATF4 activation might interfere the mutual cross-talk between ER stress and oxidative stress [20].

\section{GRP78 as an alternative receptor for SARS-CoV-2}

Several studies have shown that angiotensin-converting enzyme 2 (ACE-2) acts as the primary receptor for SARS$\mathrm{CoV}-2$ [21]. However, in certain tissues, such as endocrine cells of the prostate gland, astrocytes and pericytes in the central nervous system, and hepatocytes, there's no association between the abundancy of ACE- 2 and the severity of clinical complications [22]. Therefore, researchers have proposed alternative pathways for virus entry. Several receptors have been suggested including glucose-regulated protein 78 (GRP78), which has also been implicated in the pathogenesis of other members of the coronavirus family [23]. GRP78 is an essential chaperone in the endoplasmic reticulum (ER) and overexpressed and appears on the surface of almost all cells during stress and function as a virus receptor known as cell surface GRP78 (CS-GRP78) [24]. ER stress is triggered after rapid fluctuations in the cellular microenvironment because of different pathologies such as viral infections [24].

Considering the above-mentioned points, the components that can regulate ATF4 may be potential candidates to reduce GRP78 upregulation and it might be concluded that they can modulate ROS activation.

Chronic inflammation and/or over activity of ER stress could deteriorate the general hemostasis. In addition, oxidative stress from protein overload could impair the mitochondrial function [10]. 
Antioxidants can reduce mitochondrial ROS production by modulating ATF4 activity. Modulating ATF4 activity could moderate clinical complications of coronavirus disease 2019 (COVID-19) by cutting down GRP78 levels.

\section{GRP-78 and COVID-19 infection}

Virus glycoproteins are known the primary causes of ER stress in cells, causing unfolded protein buildup in the ER lumen and activating the UPR signaling pathway [25] that can lead to an increase in the synthesis of chaperone proteins like GRP78 [26]. The GRP78 protein has been linked to the entry of a variety of viruses [27]. The association between the SARS-CoV-2 virus and the GRP78 protein has been shown in several studies, and taking the advantage of this association, GRP78 proposed as a potential therapeutic target $[28,29]$. Several trial studies with smaller populations demonstrated that the GRP78 mRNA and protein levels were also elevated in the serum during COVID-19 infection [29, 30]. Another trial with a large statistical population revealed that GRP78 level in COVID19 patients was approximately five times higher than the healthy control group[26]. Therefore, there is a meaningful correlation between GRP-78 elevation and COVID-19 severity. Moreover, GRP78 is an alternative receptor for SARS-CoV-2 entrance and infection, and it could be a potential target for innovative clinical settings for combating different viruses that rely on GRP78 in combination therapy approaches [31].

\section{GRP78 inhibition as a potential therapeutic approach for COVID19}

GRP78 is a chaperone protein expressed in all cell types and it is believed that it could have therapeutic potential as a target for the treatment of certain diseases [32]. For example, it was shown that cancer progression associates with high level of GRP78 [33]. Some small molecules such as OSU-03012 and HA15 that are capable of suppressing GRP78 [34]. Also, some natural components can inhibit the GRP78 protein, for example, (-)-Epigallocatechin gallate (EGCG) binds to the GRP78 and acts as a competitive inhibitor of the ATPase activity of GRP78 [35]. Luteolin (3', 4', 5', 7'-tetrahydroxyflavone), a natural flavonoid produced in several plants can attenuate the up-regulation of GRP78/BiP, leading to the reduction of phospho-eIF2a, ATF4, and CHOP $[36,37]$. Salidroside ( $\mathrm{p}$-hydroxyphenethyl- $\beta$-D-glucoside) is a phenol glycoside which can reduce the expression level of GRP78/ $\mathrm{BiP}$ and other ER stress markers [38]. Lithospermic acid is another natural component that can inhibit the up-regulation of GRP78/BiP protein [39]. Some proteins such as basic fibroblast growth factor (bFGF) can inhibit the up-regulation of ER stress response-proteins in Sprague-Dawley rats. This protein inhibits the expression of GRP78/BiP and CHOP proteins [40].

\section{Conclusions}

GRP78 is an important chaperone that translocate to the surface of irritated cells and can act as a viral receptor [24]. In addition, overexpression of ATF4 plays a substantial role in the interaction between ER stress and oxidative stress. It was shown that the GRP78 level is regulated by ATF4, thus, an elevated level of ATF4 can induce GRP78. When the GRP78 protein is overexpressed under ER stress conditions, it localizes on the surface of cells and functions as a virus receptor. Therefore, blocking or inhibition of GRP78 could be a potential therapeutic strategy for COVID-19. In the present paper, we proposed a possible cross-talk between ATF4 and its potential regulatory impact on GRP78, as a potential regulator of ER stress and oxidative stress in irritated cells. GRP78 targeted therapies could open new horizons on COVID-19 critical cases (Fig. 1). 


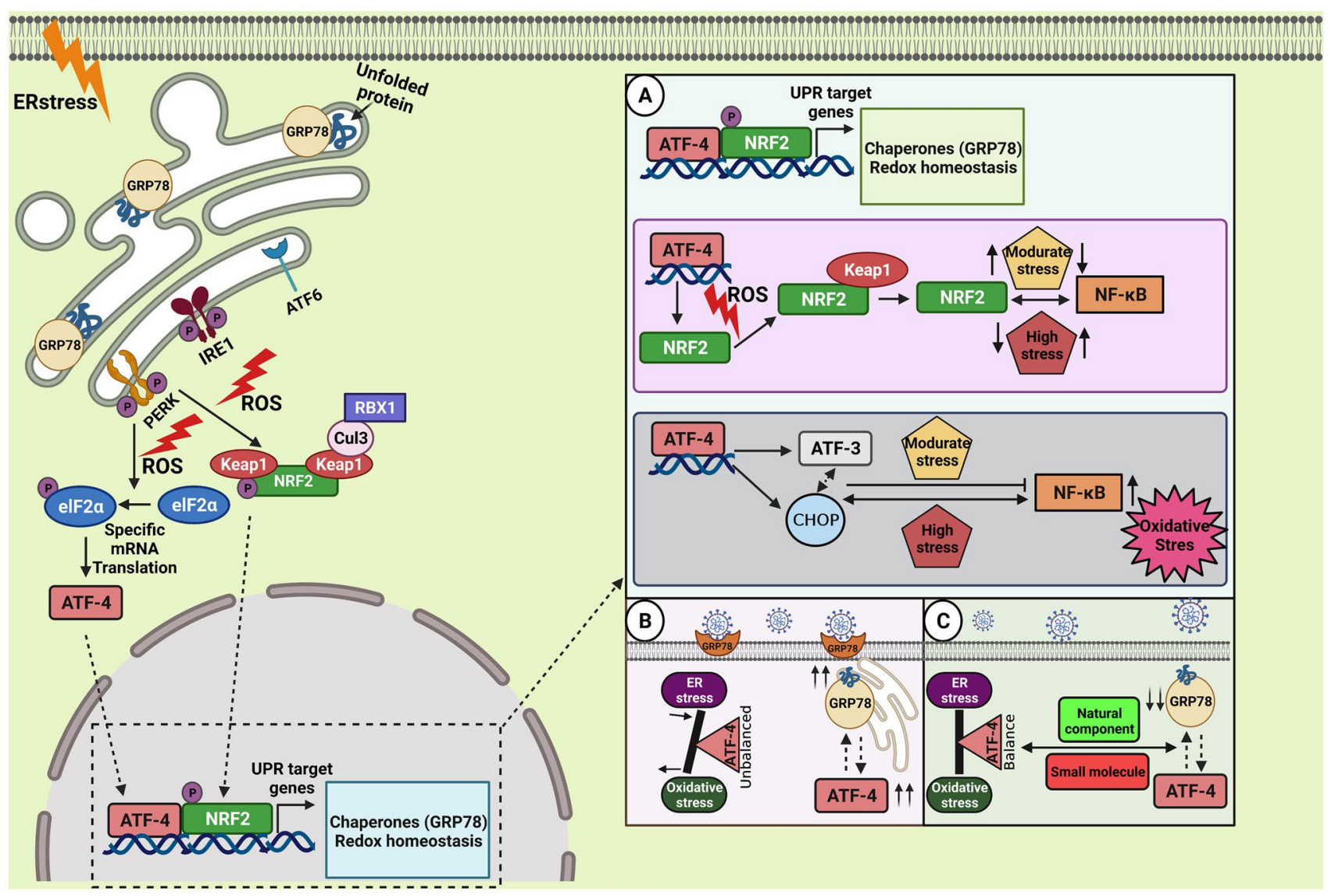

Fig. 1 The PERK kinase phosphorylates the eukaryotic initiation factor 2 (eIF2) in response to unfolded protein response in the endoplasmic reticulum (ER). When eIF2 is phosphorylated, a group of stress-responsive Transcription factors, including ATF4, translocate to the nucleus and translates more efficiently. ATF4 enhanced the production of nuclear factor-like 2 (NRF2) through the PERK pathway. While oxidative stress happens, ATF4 forms heterodimers with NRF2 to induce antioxidant transcription. A Functional cross-talk between $\mathrm{NRF} 2$ and NF- $\kappa \mathrm{B}$. The lack of NRF2 can dramatically increase $\mathrm{NF}-\kappa \mathrm{B}$ activation. Conversely, NF- $\mathrm{KB}$ can regulate NRF2 transcription and activity, in both positive and negative impacts on the target

Acknowledgements Authors appreciate supportive colleagues who helped them in different research centers to complete this work. All listed authors confirm that their research projects are supported via institutions that are primarily involved in education and/or research.

Author contributions M.S.F wrote the first draft. H.K., S.M.R.H. and M.H. involved in conceptualization and review editing, M.V involved in conceptualization, review and editing the draft and final approval.

Funding Not applicable.

\section{Declarations}

Conflict of interest The authors declare that there is no conflict of interest. gene's expression depending on the level of stress in the cells. ATF4 induces the expression of two transcription factors, ATF3 and CHOP, which usually inhibit the pathway but can activate it in stressful circumstances. B In infected cells, GRP78 level is elevated due to an increase in ER stress responses. Overexpressed GRP78 can induce ATF-4 expression. Moreover, accumulated ATF-4 synergically induces GRP-78 expression. Therefore, GRP-78 has been translocated to the cell membrane and help as an effective receptor for virus entry $\mathbf{C}$ Natural components and some small molecules can reduce ER stress through controlled GRP-78 level and ATF-4 expression

Ethical approval This article does not contain any studies with human participants or animals performed by any of the authors.

\section{References}

1. Zhu N, Zhang D, Wang W, Li X, Yang B, Song J et al (2020) A novel coronavirus from patients with Pneumonia in China, 2019. N Engl J Med 382(8):727-733

2. Fung TS, Liu DX (2014) Coronavirus infection, ER stress, apoptosis and innate immunity. Front Microbiol 5:296

3. Ron D, Walter P (2007) Signal integration in the endoplasmic reticulum unfolded protein response. Nat Rev Mol Cell Biol 8(7):519-529 
4. Alkadi HJID-DT (2020) A review on free radicals and antioxidants 20(1):16-26

5. Delgado-Roche L, Mesta F (2020) Oxidative stress as key player in severe acute respiratory syndrome coronavirus (SARS-CoV) infection. Arch Med Res 51(5):384-387

6. Mehta P, McAuley DF, Brown M, Sanchez E, Tattersall RS, Manson JJ (2020) COVID-19: consider cytokine storm syndromes and immunosuppression. The Lancet 395(10229):1033-1034

7. Varga Z, Flammer AJ, Steiger P, Haberecker M, Andermatt R, Zinkernagel AS et al (2020) Endothelial cell infection and endotheliitis in COVID-19. The Lancet 395(10234):1417-1418

8. Teijaro JR, Walsh KB, Cahalan S, Fremgen DM, Roberts E, Scott F et al (2011) Endothelial cells are central orchestrators of cytokine amplification during influenza virus infection. Cell 146(6):980-991

9. Laforge M, Elbim C, Frère C, Hémadi M, Massaad C, Nuss P et al (2020) Tissue damage from neutrophil-induced oxidative stress in COVID-19. Nat Rev Immunol 20(9):515-516

10. Chaudhari N, Talwar P, Parimisetty A, Lefebvre d'Hellencourt C, Ravanan P (2014) A molecular web: endoplasmic reticulum stress, inflammation, and oxidative stress. Front Cell Neurosci 8:213

11. Dandekar A, Mendez R, Zhang K (2015) Cross talk between ER stress, oxidative stress, and inflammation in health and disease. Methods in molecular biology (Clifton, NJ). ;1292:205-14

12. Wortel IMN, van der Meer LT, Kilberg MS, van Leeuwen FN (2017) Surviving stress: modulation of ATF4-mediated stress responses in normal and malignant cells. Trends Endocrinol Metab 28(11):794-806

13. Bhandary B, Marahatta A, Kim HR, Chae HJ (2012) An involvement of oxidative stress in endoplasmic reticulum stress and its associated diseases. Int J Mol Sci 14(1):434-456

14. Szegezdi E, Logue SE, Gorman AM, Samali A (2006) Mediators of endoplasmic reticulum stress-induced apoptosis. EMBO Rep 7(9):880-885

15. Bhattarai KR, Riaz TA, Kim HR, Chae HJ (2021) The aftermath of the interplay between the endoplasmic reticulum stress response and redox signaling. Exp Mol Med 53(2):151-167

16. Zong Y, Feng S, Cheng J, Yu C, Lu G (2017) Up-regulated ATF4 expression increases cell sensitivity to apoptosis in response to radiation. Cell Physiol Biochem Int J Exp Cell Physiol Biochem Pharmacol 41:784-7942

17. Kasai S, Shimizu S, Tatara Y, Mimura J, Itoh K (2020) Regulation of Nrf2 by mitochondrial reactive oxygen species in physiology and pathology. Biomolecules. 10(2):320

18. Wang C, Li H, Meng Q, Du Y, Xiao F, Zhang Q et al (2014) ATF4 deficiency protects hepatocytes from oxidative stress via inhibiting CYP2E1 expression. J Cell Mol Med 18(1):80-90

19. Xia S, Duan W, Liu W, Zhang X, Wang Q (2021) GRP78 in lung cancer. J Transl Med 19(1):118

20. Luo S, Baumeister P, Yang S, Abcouwer SF, Lee AS (2003) Induction of Grp78/BiP by translational block: activation of the Grp78 promoter by ATF4 through and upstream ATF/CRE site independent of the endoplasmic reticulum stress elements. J Biol Chem 278(39):37375-37385

21. Bian J, Li Z (2021) Angiotensin-converting enzyme 2 (ACE2): SARS-CoV-2 receptor and RAS modulator. Acta pharmaceutica Sinica B 11(1):1-12

22. Singh M, Bansal V, Feschotte C (2020) A single-cell RNA expression map of human coronavirus entry factors. bioRxiv: the preprint server for biology.

23. Vankadari N, Wilce JA (2020) Emerging WuHan (COVID-19) coronavirus: glycan shield and structure prediction of spike glycoprotein and its interaction with human CD26. Emer Micro Infections 9(1):601-604
24. Shahriari Felordi Mahtab MA, Najimi, Mustapha (2021) Vosough massoud. Is there any alternative receptor for SARS-CoV-2? Cell J 23(2):247-250

25. Versteeg GA, van de Nes PS, Bredenbeek PJ, Spaan WJ (2007) The coronavirus spike protein induces endoplasmic reticulum stress and upregulation of intracellular chemokine mRNA concentrations. J Virol 81(20):10981-10990

26. Sabirli R, Koseler A, Goren T, Turkcuer I, Kurt OJLS (2021) High GRP78 levels in Covid-19 infection: A case-control study. 265:118781

27. Chu H, Chan CM, Zhang X, Wang Y, Yuan S, Zhou J et al (2018) Middle East respiratory syndrome coronavirus and bat coronavirus HKU9 both can utilize GRP78 for attachment onto host cells. J Biol Chem 293(30):11709-11726

28. Ibrahim IM, Abdelmalek DH, Elshahat ME, Elfiky AA (2020) COVID-19 spike-host cell receptor GRP78 binding site prediction. J Infect 80(5):554-562

29. Köseler A, Sabirli R, Gören T, Türkçüer I, Kurt Ö (2020) Endoplasmic reticulum stress markers in SARS-COV-2 infection and Pneumonia: case-control study. In vivo (Athens, Greece) 34(3 Suppl): $1645-50$

30. Palmeira A, Sousa E, Köseler A, Sabirli R, Gören T, Türkçüer İ et al (2020) Preliminary virtual screening studies to identify GRP78 inhibitors which may interfere with SARS-CoV-2 infection. Pharmaceuticals (Basel, Switzerland). 13(6):132

31. Carlos AJ, Ha DP, Yeh D-W, Van Krieken R, Tseng C-C, Zhang $P$ et al (2021) The chaperone GRP78 is a host auxiliary factor for SARS-CoV-2 and GRP78 depleting antibody blocks viral entry and infection. J Biol Chem 296:100759

32. Zhu X, Zhang J, Fan W, Wang F, Yao H, Wang Z et al (2013) The rs391957 variant cis-regulating oncogene GRP78 expression contributes to the risk of hepatocellular carcinoma. Carcinogenesis 34(6): 1273-1280

33. Lee AS (2014) Glucose-regulated proteins in cancer: molecular mechanisms and therapeutic potential. Nat Rev Cancer 14(4):263-276

34. Booth L, Roberts JL, Cash DR, Tavallai S, Jean S, Fidanza A et al (2015) GRP78/BiP/HSPA5/Dna K is a universal therapeutic target for human disease. J Cell Physiol 230(7):1661-1676

35. Ermakova SP, Kang BS, Choi BY, Choi HS, Schuster TF, Ma W-Y et al (2006) (-)- Epigallocatechin gallate overcomes resistance to etoposide-induced cell death by targeting the molecular chaperone glucose-regulated protein 78. Cancer Res 66(18):9260-9269

36. Nabavi SF, Braidy N, Gortzi O, Sobarzo-Sanchez E, Daglia M, Skalicka-Woźniak K et al (2015) Luteolin as an anti-inflammatory and neuroprotective agent: A brief review. Brain Res Bull 119:1-11

37. Hu L-W, Yen J-H, Shen Y-T, Wu K-Y, Wu M-J (2014) Luteolin modulates 6-hydroxydopamine-induced transcriptional changes of stress response pathways in PC12 cells. PLoS ONE 9(5):e97880

38. Tao K, Wang B, Feng D, Zhang W, Lu F, Lai J et al (2016) Salidroside protects against 6-hydroxydopamine-induced cytotoxicity by attenuating ER stress. Neurosci Bull 32(1):61-69

39. Lin Y-L, Tsay H-J, Lai T-H, Tzeng T-T, Shiao Y-J (2015) Lithospermic acid attenuates 1-methyl-4-phenylpyridine-induced neurotoxicity by blocking neuronal apoptotic and neuroinflammatory pathways. J Biomed Sci 22(1):1-13

40. Cai P, Ye J, Zhu J, Liu D, Chen D, Wei X et al (2016) Inhibition of endoplasmic reticulum stress is involved in the neuroprotective effect of bFGF in the 6-OHDA-induced Parkinson's disease model. Aging Disease 7(4):336

Publisher's Note Springer Nature remains neutral with regard to jurisdictional claims in published maps and institutional affiliations. 